\title{
Pervaporative transfer modeling through dense membranes validation of the scattered packet method
}

\author{
A. Essamri ${ }^{1}$, R. Elgouri ${ }^{2,3}$, F.Z. Zouair ${ }^{1}$, N. Cheikhi ${ }^{1}$, L. Hlou ${ }^{2}$ \\ ${ }^{1}$ Laboratory of Agroressources and process engineering, Faculty of Sciences, University Ibn Tofail, Kenitra, Morocco \\ ${ }^{2}$ Laboratory of Electrical Engineering and Energy Systems, Faculty of Sciences, University Ibn Tofail, Kenitra, Morocco \\ ${ }^{3}$ National School of Applied Sciences (ENSA), Ibn Tofail University, Kenitra, Morocco \\ *Corresponding author E-mail: essamri@gmail.com
}

\begin{abstract}
The study of the diffusion through a homogeneous dense film, covers practically and theoretically a fundamental interest. In pervaporation (membrane separation process) the diffusion is the limiting step in the operation of the transfer and thus directly influences the performance of the membrane. The knowledge of the diffusion coefficient of an aqueous solution in a membrane makes it possible to elucidate the mechanism of this elementary step of pervaporation. Several methods were used to determine the diffusivity of a compound through a dense membrane (differential permeation, kinetic of sorption).

In this work, we tried to validate the method of the scattered packet. It is about a digital method, of cellular automaton, which produces really the process of diffusion through a membrane using the second law of Fick in which the diffusion coefficient is considered constant. Permeation flux data obtained by simulation allowed validating this method. By using the method of relaxation time and the method of maximum slope, can thus determine the coefficient of diffusion of diffusing species through the membrane.
\end{abstract}

Keywords: Diffusivity, Membrane, Pervaporation, Scattered Packet Method.

\section{Introduction}

Pervaporation is a membrane technique, which combines the partial evaporation of the constituents of a liquid mixture with their selective permeation through a dense membrane (Neel \& Aptel 1982, Brun 1980). It is currently the object of intensive research, these regarding both the development of efficient membranes, the theory of transfer through these membranes and the optimization of the process.

To elucidate the mechanism of the pervaporatif transfer, several studies were carried out and the proposed models are in general very complex. However most authors agree on the three steps of the mechanism of pervaporation: sorption of molecules in the membrane at the upstream face, diffusion of these molecules through the membrane and finally their desorption in vapor phase (Long 1965, Fels \&Huang 1971).

Under our experimental conditions where the pressure downstream of the membrane is often maintained very low, the step of desorption is considered very fast and non-selective (Rautenbach $\&$ Albercht 1989). The diffusion within the membrane is thus the limiting step of the mass transfer. As a result, the study of the diffusion parameter, governing the permeation of a species through a polymeric film, takes on a fundamental interest.

Many digital models were worked out to determine the diffusion coefficient, from the result of material flow in transitory mode. These models are based on analytical expressions weighted by parameters (Stern \& Saxena 1980, Mulder \& Smolders 1984, Mulder et al. 1985).
In this work we used a new digital method, a cellular automaton was developed which actually reproduces the process of diffusion through a membrane by simulating the real evolution of the scattering particles through the polymeric film: the scattered packet method (SPM).

The work presented in this paper is to study the material flow in transitory mode, in order to determine the diffusion coefficient, using both the analytical method and the new digital method of scattered packet.

\section{Analytical method}

This method consists in determining the flow of permeation in transitory mode from the resolution of the second Fick's law, which can be written for a flat membrane in the following form:

$$
\frac{\delta C_{i(t, x)}}{\delta t}=\frac{\delta}{\delta x}\left(\mathrm{D}_{\mathrm{i}} \frac{\delta C_{i(t, x)}}{\delta x}\right)
$$

This law, in which the diffusion coefficient is constant, admits many analytical solutions which depend on the initial conditions and the limitations that it attaches:

$\mathrm{C}_{\mathrm{i}}(0, \mathrm{x})=0, \mathrm{C}_{\mathrm{i}}(\mathrm{t}, 0)=\mathrm{C}_{\text {io }}$ et $\mathrm{C}_{\mathrm{i}}(\mathrm{t}, \mathrm{l})=0$

Under these conditions, the transmembrane flow $\mathrm{J}$ of

A species i can be described (Barrer 1941, Palmai \& Olah 1984) by:

$$
\mathrm{J}_{\mathrm{i}}=\frac{D_{i} A C_{i}}{l}(1+2) \sum_{n=1}^{\infty}\left((-1)^{n} \exp \left(\frac{-n^{2} \Pi^{2} D_{i} t}{l^{2}}\right)\right)
$$


Where Di is the diffusion coefficient of the compound $i, C_{i}$ is its concentration at the upstream

Side, where A is the surface of the membrane, 1 is the thickness and $t$ is the time.

\section{Scattered packet method}

The method of the scattered packet is a new microscopic method, developed by L.Hlou (Hlou 2003). It was applied to the resolution of the Boltzmann equation for the study of the evolution of the electrons in the semiconductor (Hlou 1985, Vaissiere 1986). This method is based on the simulation of a package of particles which evolves in a field of study. We will apply it to the study of diffusion through a membrane.

The operation is to subdivide the membrane in rectangular cells of thickness $\Delta \mathrm{x}$ (figure 1). Each cell is identified by an index $\mathrm{i}$ of space and the whole of particles it contains at a given moment constitutes a package. If 1 is the thickness of the membrane, the number of cells along direction ox is obviously $\mathrm{N}_{\mathrm{x}}=\mathrm{l} / \Delta \mathrm{x}$. The problem is therefore to know at any moment, the distribution of these packages in the reporting membrane and consequently, the concentration of particles in each cell i.

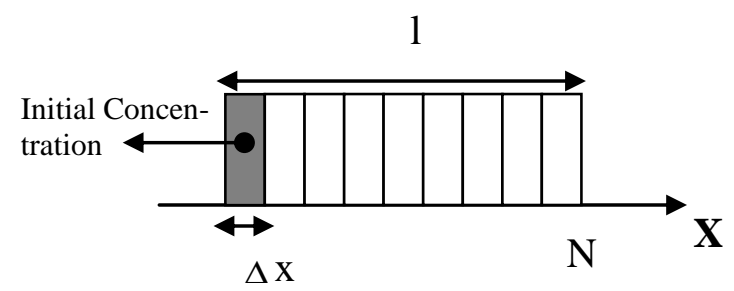

Fig. 1: Initial Distribution of the Concentration

Indeed, this (SPM) method allows appreciating the change in the concentration of the load during the experiment, which is not the case for (MA) which requires the use of a rather large volume so that the variation of concentration is negligible.

In addition this method (SPM) can be applied for all membranes shapes, which is not possible for the (MA) which requires a flat or cylindrical shape.

\section{Determination of transitory flow}

To determine the flow of permeation $\mathrm{J}$ at a moment $\mathrm{t}+\Delta \mathrm{t}$, knowing concentration $\mathrm{C}_{\mathrm{i}}$ at the moment $\mathrm{t}$, we use the expression: $\mathrm{J}=\mathrm{C}$. $\mathrm{V}_{\mathrm{d}}$

Where $V_{d}$ is the diffusion rate obtained by identification of the equation (3) with the first law of Fick, known by the formula:

$\mathrm{J}=-\mathrm{D} \nabla C=-\mathrm{D} \frac{\partial C}{\partial x}$

Indeed, the expression of the rate of diffusion is:

$\mathrm{V}_{\mathrm{d}}=-\frac{D}{C} \frac{d C}{d x}$

As a result of the diffusion, during a time interval $\Delta \mathrm{t}$, the particles of cell i move at the speed $V_{d}$ towards the neighbouring cell. Thus, the content of each cell of arrival is enriched by a quantity of particles, proportional to the recovery which it has with the incidental package.

The study of the transitional mode is brought back thus, with a simple calculation of concentration of particles arriving and leaving a package given at a time t. Indeed, starting with a $\mathrm{C}$ (i) concentration in a cell $i$, known at the moment $t$, the concentration of this same package at the moment $\mathrm{t}+\Delta \mathrm{t}$ is:

$\mathrm{C}_{\mathrm{i}}(\mathrm{t}+\Delta \mathrm{t})=\mathrm{C}_{\mathrm{i}}(\mathrm{t})+\mathrm{C}_{\mathrm{i}}^{(\mathrm{e})}(\mathrm{t})-\mathrm{C}_{\mathrm{i}}^{(\mathrm{s})}(\mathrm{t})$

$C_{i}{ }^{(e)}$ is the term of entry in cell $i$, it presents the concentration of particles that arrive in cell $i$, from the cell $i-1$.

$\mathrm{C}_{\mathrm{i}}^{(\mathrm{s})}$ is the term of outlet in the cell $\mathrm{i}$, it represents the concentration of the particles leaving the cell $i$ to cell $i+1$.
Moreover, the concentration of particles that remains in the cell $\mathrm{i}$ am represented by:

$\mathrm{C}_{\mathrm{i}}^{(\mathrm{r})}(\mathrm{t})=\mathrm{C}_{\mathrm{i}}(\mathrm{t})-\mathrm{C}_{\mathrm{i}}^{(\mathrm{s})}(\mathrm{t})$

This remained concentration is defined by the difference $(\mathrm{x}-\delta)$, where $\Delta \mathrm{x}$ is the thickness of each cell, $\delta$ being the elementary movement of each package during a time interval $\Delta \mathrm{t}$, it is defined as the product of speed $\mathrm{Vi}$ of particles in package $i$, by $\Delta \mathrm{t}$. It is supposed that thickness $\Delta \mathrm{x}$ of the cell is small enough to consider that the particles in each cell $i$ would have the same speed $V_{I}$.

In addition, $\Delta \mathrm{t}$ is chosen small enough, so that covering between two neighbouring cells is partial, which means that it must take a $\Delta \mathrm{t}$ such as:

$\mathrm{V}_{\max } \Delta \mathrm{t}<\Delta \mathrm{x}$

Under these conditions, we can schematize the elementary displacement of a cell i during a time interval $\Delta \mathrm{t}$, by the following figure:

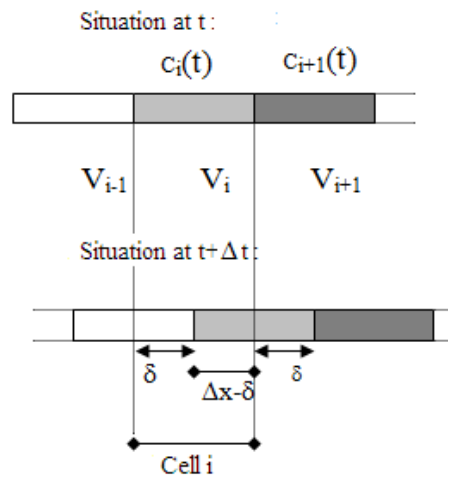

Fig. 2: The Displacement Result of a Packet.

The flow chart of the simulation program of diffusion of a particle through a dense membrane is presented in appendice A.

\section{Results and discussion}

The permeation flow simulation of a component through a membrane was carried out using two methods:

- The analytical method (AM): it is based on mathematical expression of flow according to time, limited by the initial conditions and the limits, constraints and non - homogeneity of the membrane.

- The new method of scattered packet (SPM) which has the advantage of the real simulation of the diffusion phenomenon regardless of the complexity of the system to be studied (nonconstant coefficient of diffusion and concentration at the level of the downstream side of the membrane).

\subsection{Permeation flux}

We represented on figure 3 the paces of curves of the flux variation at various times, found by the two methods (AM and SPM). We took into account the assumptions of the (AM) which requires constant concentration and diffusion coefficient of penetrating through a flat membrane. We considered that the concentration is constant in the upstream side and worthless at the downstream side of the membrane 


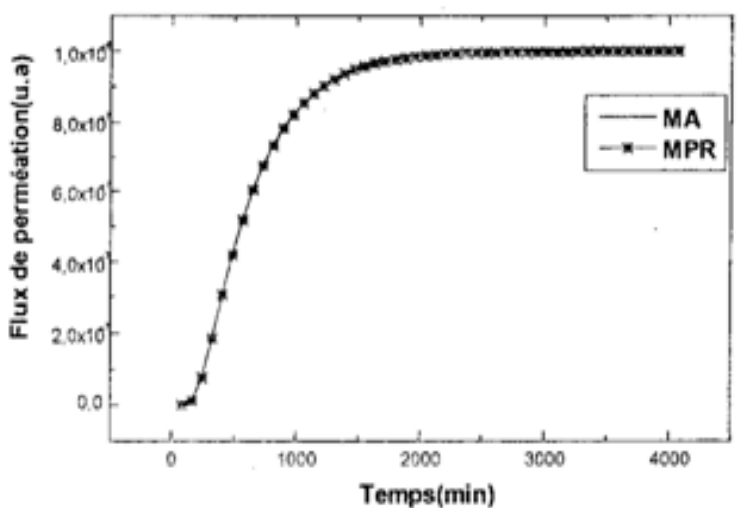

Fig. 3: Comparison of Two Methods of Simulation of Permeation Flow through a Membrane.

SPM: Scattered Packet Method. MA: Analytical Method.

Parametrs used: $\mathrm{C}_{0}=100$ mole $/ \mathrm{l}, \mathrm{D}=5.4510^{-13} \mathrm{~m}^{2} / \mathrm{s}, \mathrm{l}=3510^{-6} \mathrm{~m}$ This figure shows a perfect agreement between the analytical method and the scattered packet method. Both show that permeation flux start by growing (Transitory Mode of Diffusion) to reach as from a time threshold, a plate: this is the stationary mode.

It should be noted that the (SPM) is a more general method; it can not only consider these simple systems, but also the more complex situations which appear at the time of a constraint or the variation of concentration or the coefficient diffusion (Composite Membranes). Similarly this method can be applied for various geometry barriers, operating under different limiting conditions.

\subsection{Concentration profile}

The (SPM) method allows us, moreover, to provide the profile of the concentration inside the membrane. The result is illustrated in figure 4 .

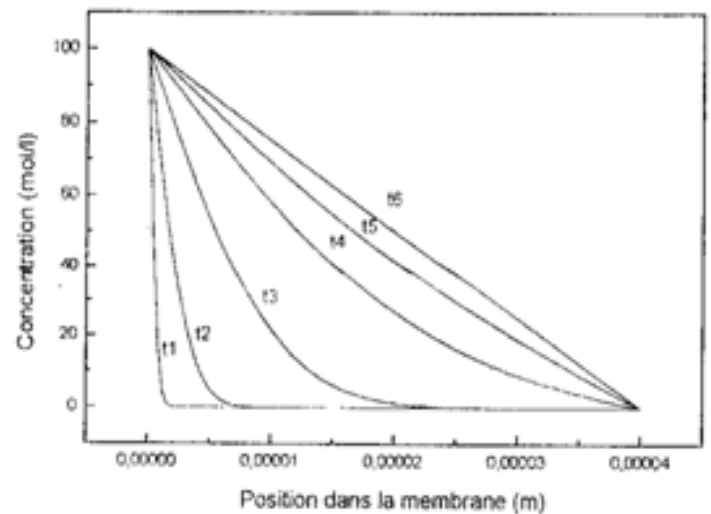

Fig. 4: Profile of Concentration inside the Membrane, Calculated By SPM for Various Times.

Parameters used: $\mathrm{C}_{0}=100$ mole $/ \mathrm{l}, \mathrm{D}=5.4510^{-13} \mathrm{~m}^{2} / \mathrm{s}$ and $\mathrm{l}=3510^{-6}$ $\mathrm{m}$

We note a damping of the concentration over time within the membrane. This comes from the progressive migration of particles from penetrating of the upstream side of the membrane $(\mathrm{x}=0)$, of concentration $\mathrm{C}=100 \mathrm{~mol} / \mathrm{l}$, towards the downstream side $(\mathrm{x}=1)$ initially devoid of particles (concentration zero). Indeed, there is much enrichment, over time,

From the downstream side of a quantity of particles that reaches $12 \mathrm{~mol} / \mathrm{l}$ at time $\mathrm{t}_{6}$.

This behavior can be explained by the Brownian movement which sends particles in all directions of space. However in the presence of a concentration gradient, this random movement leads to a transfer of particles of the concentrated regions to the more dilute. A comment further in figure 4 shows that curves tend to become linear over time, until observation of a straight line at time $\mathrm{t}_{6}$. This time can only be time threshold corresponding to the transition from the transitory mode towards the stationary mode.

Indeed, in stationary mode flow is constant in all the thickness of the membrane, which imposes the constant concentration gradient: $\mathrm{J}=-\mathrm{D} \mathrm{dc} / \mathrm{dx}$ et $\mathrm{Dc} / \mathrm{dx}=$ constante

This expression shows that the concentration varies linearly with the distance $\mathrm{x}$ traversed by the diffusing species, which is compatible with the line shown in figure 4 at time $t_{6}$.

\subsection{Determination of diffusion coefficient}

Several methods were used for the determination of diffusion coefficient of a solvent through a membrane:

\subsubsection{Method of $t 1 / 2$}

It is to determine the time $t_{1 / 2}$ at the end of which the flow of permeation reaches half of its value in stationary mode $\mathrm{J}_{0}$. The relation linking $\mathrm{D}$ at this time is deduced from the development of the following equation (Ziegel et al. 1969):

$J=\frac{D A C}{l}\left(1+2 \sum_{n=1}^{\infty}\left[(-1)^{n} \exp \left(\frac{-n^{2} \Pi^{2} D t}{l^{2}}\right)\right]\right.$

1: Thickness of the membrane,

A: The membrane surface,

C: Concentration of the diffusing species.

Thus for a flow of permeation equal to half of stationary permeation flux, the diffusion coefficient is given by the relation:

$\mathrm{D}=1^{2} / 7.199 \mathrm{t}_{1 / 2}$

\subsubsection{Analytical method}

This method is based on the resolution of the second law of Fick. The theoretical curve smoothing, obtained by simulation of this equation, and the experimental results allows to deduce the diffusion coefficient by using the Rosenbrok algorithm (Uchtil et al. 1996).

\subsubsection{Scattered packet method (SPM)}

In the context of the (SPM), we used two different ways to determine the diffusion coefficient of a solvent through the membrane.

\subsubsection{Method of relaxation time}

It is based on the extraction of relaxation time necessary to pass from a transitory time $t_{1 / 10}$ a quasi-stationary time $t_{9 / 10}$, after which the flow reaches values equal to $1 / 10$ and $9 / 10$ of the value obtained in stationary mode. Where as

The non-dimensionalized equation, deduced from equation (I.1):

$$
J_{D, l}^{\prime}(t)=1+2 \sum_{n=1}^{\infty}\left[(-1)^{n} \exp \left(\frac{-n^{2} \Pi^{2} D_{i} t}{l^{2}}\right)\right]
$$

With: $\mathrm{J}_{\mathrm{D}, 1}=\mathrm{J} / \mathrm{J}_{0}$

Noting that the flow of permeation $J^{\prime}{ }_{D / 1}(t)$ depends only on thickness 1 , the coefficient of diffusion $\mathrm{D}$ and time t. So when considering a change in variable which gathers these three parameters in the same variable $t$ ', there will be:

$$
J^{\prime}\left(t^{\prime}\right)=1+2 \sum_{n=1}^{\infty}\left[(-1)^{n} \exp \left(-n^{2} \Pi^{2} t^{\prime}\right)\right]
$$

In this implicit writing, the flow $\mathrm{J}$ ' is independent of $\mathrm{D}$ and 1 , this appears clearly in figure 5, which represents J' according to the new variable $t$ ', for different values of $D$ and 1 . It gets the same curve for different cases. In addition, it can be seen that the value of the difference between t' ${ }_{9 / 10}$ and $t^{\prime}{ }_{1 / 10}$ is a constant that we note Cs:

$\mathrm{C}_{\mathrm{s}}=\mathrm{t}^{\prime}{ }_{9 / 10}-\mathrm{t}^{\prime}{ }_{1 / 10}$ 


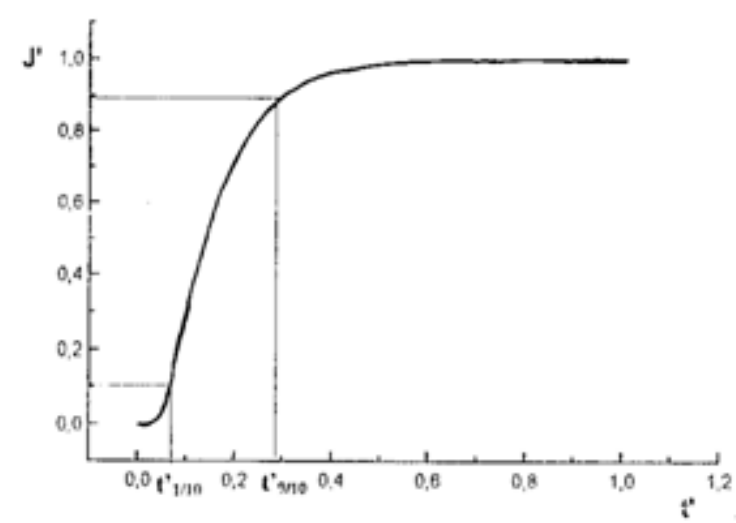

Fig. 5: Representation of the Curves of Non-Dimensionalized Flow J' For Various Values of Coefficient of Diffusion and Thickness.

Using this property, we can deduce geometrically the diffusion coefficient of a membrane of thickness 1 , by simple extraction of the relaxation time $\tau$ that is:

$\tau=\mathrm{t}^{\prime}{ }_{9 / 10}-\mathrm{t}^{\prime}{ }_{1 / 10}$

Replacing t' with its value in the equation (13):

$C_{S}=\frac{D}{l^{2}}\left(t_{9 / 10}-t_{1 / 10}\right)$

\subsubsection{Method of maximum slope}

It initially consists in plotting a calibration curve presenting the maximum slopes, of the flux variation according to time, for different values of coefficient of diffusion D and thickness 1. Figure 6 shows the results.
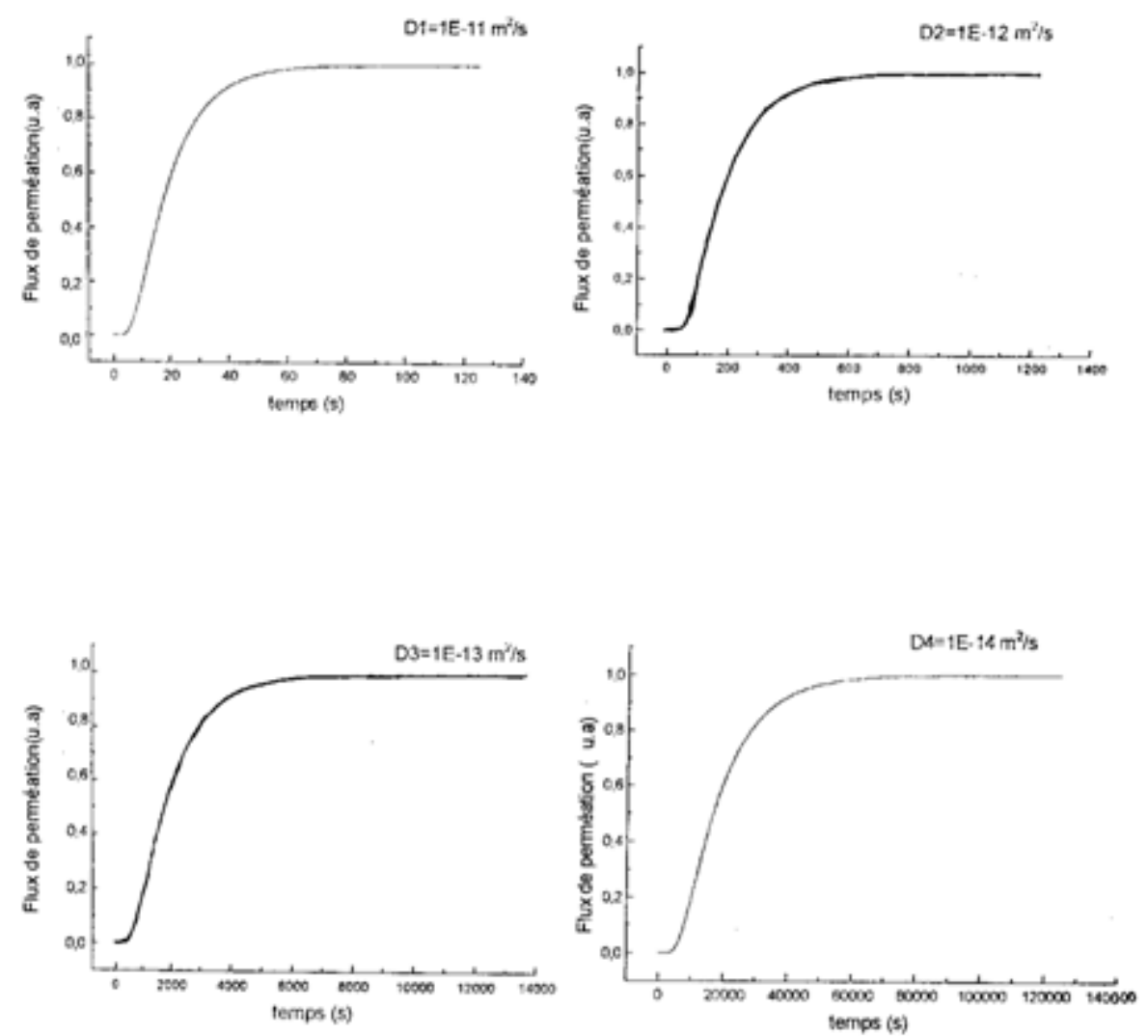

Fig. 7: Permeation Flux Obtained by Simulation, for Four Values of Diffusion Coefficient.
These results of flow allow us to validate the development of our programs, by calculating the coefficient of diffusion by each method, and then comparing it with that of departure (initialized in the

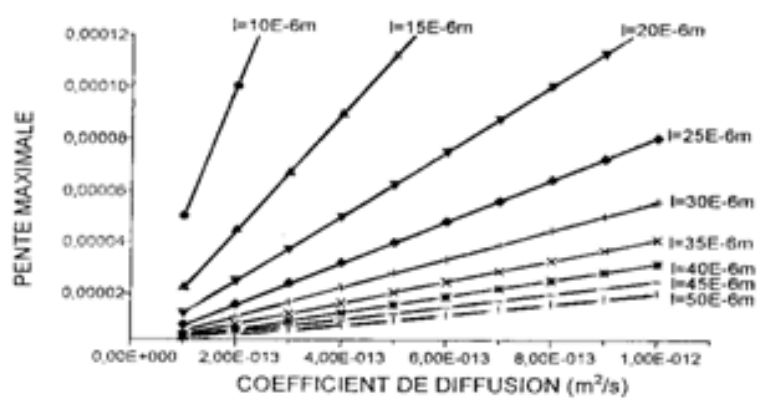

Fig. 6: Variation of the Maximum Slope According To the Diffusion Coefficient for Various Values of Membrane Thickness.

Then, the relation who links $\mathrm{D}$ to $\tau$ is in the following form:

$D=\frac{C_{s} l^{2}}{\tau}$

Thus, knowing the maximum slope of the experimental curve and referring to the calibration curve obtained by simulation, we can easily reach the diffusion coefficient.

To validate the new methods intended for the determination of diffusion coefficient, we used the data of permeation flux obtained by simulation, for four Values of diffusion coefficient, figure 7 .
Used flow simulation). We applied in addition to the method of relaxation time and the maximum slope method we have developed, the t1/2 method is the most commonly used. The results are gathered in table 1 . 
Table 1: Coefficient of Diffusion Obtained by the Method of $T_{1 / 2}$ the Method of Relaxation Time and Method of Maximum Slope.

\begin{tabular}{llll}
\hline \multicolumn{3}{c}{ Coefficient of diffusion $\left(\mathrm{m}^{2} / \mathrm{s}\right)$} \\
\hline $\mathrm{D}$ initial & $\mathrm{D}\left(\mathrm{t}_{1 / 2}\right)$ & $\mathrm{D}(\tau)$ & $\mathrm{D}(\max$ slope. $)$ \\
$1.10^{-14}$ & $1.0011910^{-14}$ & $0.9949610^{-14}$ & $1.10^{-14}$ \\
$1.10^{-13}$ & $1.0011910^{-13}$ & $0.9949610^{-13}$ & $1.10^{-13}$ \\
$1.10^{-12}$ & $1.0011910^{-12}$ & $0.9949610^{-13}$ & $1.10^{-12}$ \\
$1.10^{-11}$ & $1.0011910^{-11}$ & $0.9949610^{-11}$ & $1.10^{-11}$ \\
\hline
\end{tabular}

D initial: Coefficient of diffusion initialized in the simulation of flux in $\mathrm{m}^{2} / \mathrm{s}$.

We note that the three methods give good performances: the coefficients of diffusion obtained are

Comparable to those initialized at the beginning in simulations of flows.

We have therefore, for the study of the permeation of a compound through a dense membrane, a new

Method: method of scattered packet. It allows to determine the coefficient of diffusion of this compound and to predict its concentration profile inside this film.

\section{Conclusion}

Pervaporation is a separation technique where the driving force is generated by the gradient of activity or chemical potential of a species between the two sides of the membrane. This process includes three successive steps: sorption, diffusion and desorption However the molecular diffusion is the slowest of these steps and it is therefore kinetically determining.

Thus, we were interested in this work at the step of diffusion (case of flat membranes). We tried to model using two methods:

- The analytical method (AM), the most commonly used, it is based on the permeation flux equation, obtained from the resolution of the second law of Fick.

- The digital method, so-called scattered packet method (SPM), used for the first time for the case of membranes. This method allows simulating the real evolution of the particles through a polymeric film, which allows us to predict the path of the scattering particles inside this film. In addition, it can be applied to various shapes of membranes (flat, cylindrical, spherical...), while the analytical method treats only the case of the flat or cylindrical membranes.

The results of simulation of permeation flow obtained show a perfect agreement between the two methods.

Also the knowledge of the value of the coefficient of diffusion of a solute in a membrane takes a fundamental interest. In order to determine this parameter, we developed two new methods: the method of maximum slope and the method of relaxation from the (SPM) time.

To validate these methods, we used the flow data obtained by simulation. We determined the diffusion coefficient by these two new methods and a method already used which is the $t_{1 / 2}$ method. We found that the values of the diffusion coefficients obtained are comparable to those are initialized for the used flow simulation.

\section{References}

[1] Neel J \& Aptel P (1982), La pervaporation, Première partie: Principe de la technique Entropie, Vol.54, No. 104.

[2] Brun JY.P (1980), Procédés de séparation par mem-branes, Edition Masson.

[3] Long R.B (1965), Liquid permeation through plastic film, Ind. Eng. Chem, Vol.45, 53

[4] Fels M \&Huang Y. M (1971), Theoretical interpreta-tion of the effet of mixture composition on separation of liquid in polymers, Journal of $\begin{array}{llll}\text { Macromol. } & \text { Sci. } & \text { Phys, } & \text { Vol.5, }\end{array}$ http://dx.doi.org/10.1080/00222347108212522.

[5] Rautenbach R \& Albercht R (1989), Membranes pro-cess, Edit. J. Wiley \& sons.

[6] Stern S \& Saxena Y (1980), Concentration-dependent transport of gases and vapors in glassy polymers, J. Memb. Sci, Vol. 7, 47-59. http://dx.doi.org/10.1016/S0376-7388 (00)83184-1.
[7] Mulder V \& Smolders C (1984), on mehcanisme of separation mixtures by pervaporation: calculation of concentration profiles, J. Memb. Sci, Vol.17, 289. http://dx.doi.org/10.1016/S0376-7388 (00)83220-2.

[8] Mulder V, Franken a \& Smolders C (1985), on mehca-nisme of separation mixtures by pervaporation: Experi-mental concentration profiles, J. Memb. Sci, Vol.23, 4. http://dx.doi.org/10.1016/S0376-7388 (00)83133-6.

[9] Barrer R.M (1941), Diffuson in and through solids Cambridge University, Press London.

[10]Palmai G \& Olah K (1984), new differential permea-tio rate methode for determination of membrane transport parameteres of gases, J.Memb.Sci, Vol.21, pp. 161. http://dx.doi.org/10.1016/S0376-7388 (00)81552-5

[11]Hlou L Thèse de doctorat (2003), Montpellier France.

[12]Hlou L Thèse de doctorat 3eme cycle (1985) Montpel-lier France.

[13] Vaissiere J.C (1986), Etude du régime de porteurs chauds dans le silicium de type $P$, Thèse de doctorat d'Etat, Montpellier France.

[14]Ziegel D.K. K.Frensdorft H \& Blair D.E (1969), Measurement of hydrogen isotope transport in poly (vinyl fluoride) films by the permea-tion-rate method, J.Polym.Sci, Vol.7, No.5, 809

[15]Uchtil P, Nguyen Q.T, Clement R, Grosse J \& Essamri A(1996), Diffusion of acetic acid through PVA me-mebranes. Coupling effets. Polymer, Vol.37, 93-100. http://dx.doi.org/10.1016/0032-3861 (96)81603-X

\section{Appendice}

a) Flow chart of simulation of the diffusion of particle through a dense membrane.

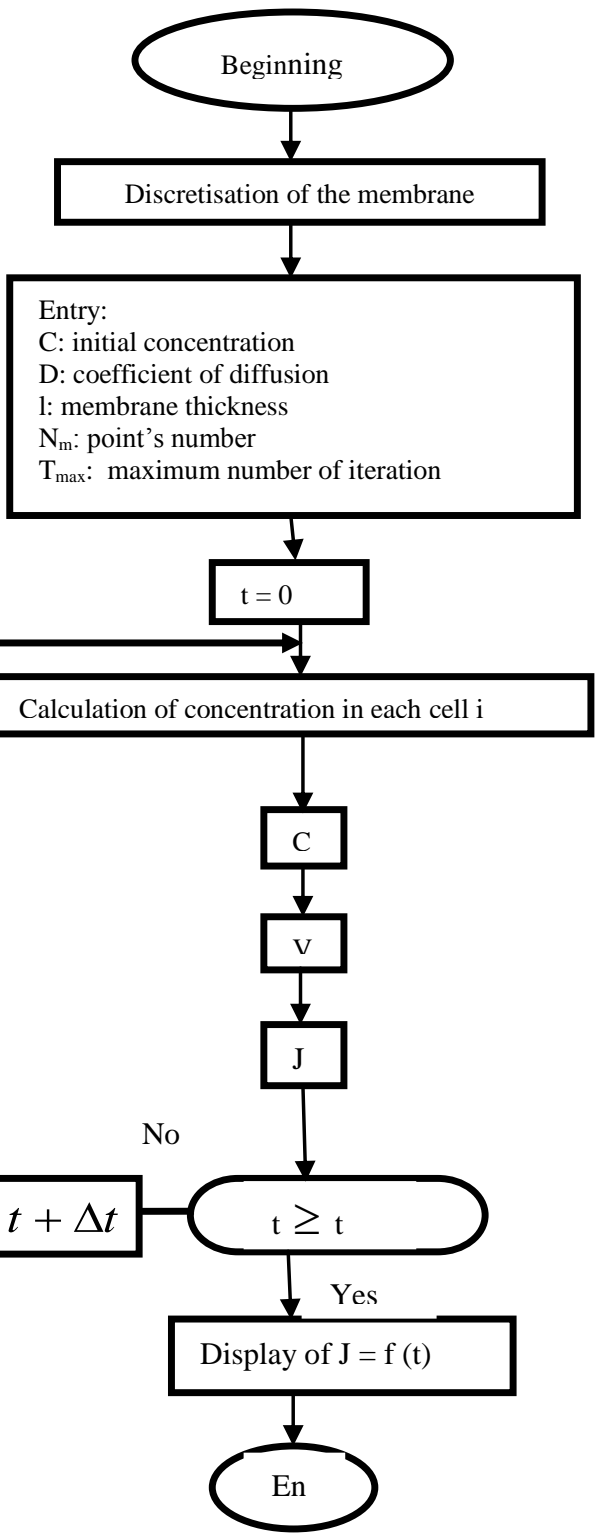

